correct application of the Mental Health Act 1983, the multidisciplinary team approach to patient management, after-care programmes and the intricacies of the referral system. We had to rely on others for the implementation of the Act. However, in addition to the initial shadowing the Mersey Care NHS Trust provided further assistance by appointing a mentor with whom we could discuss our professional difficulties. The trust also provided assistance for the Section 12 course approval, which is essential for application of the Mental Health Act 1983. For those of us who are not yet Section 12 approved, we are constantly supervised and assisted by another senior consultant.

\section{Conclusions}

Moving to the UK was not only a professional challenge for us but also a testing time for our families. There were issues related to living in a new country with the different lifestyle, food and educational system. For some of us, it was a matter of supporting our children who experienced a culture shock; for others, safety and security on the streets were major concerns.

One of the major factors that has helped us to settle down here has been the support we have drawn from each other. Those who came here earlier provided excellent assistance and guidance to the newcomers. We frequently interact with each other, make suggestions and put others at ease. The fact that few of us knew each other before arriving in the UK only helped the situation further. Our colleagues in our home countries have reacted variably to our move. Although there was some concern at the potential loss of trained practitioners (especially those who were in government jobs), we met with a general feeling of acceptance of our move. We have often discussed with our colleagues back home the similarities and differences in health systems and the challenges of working in the NHS.

Overall moving has been a challenging experience. We still feel anxious, nervous and lonely at times, and miss our families. At other times we feel at ease.

It would be immensely helpful if future programmes included a more comprehensive induction programme focusing on the structure of the NHS, the Mental Health Act 1983 and the city where recruits would work. Some knowledge by existing staff about the culture of overseas doctors and their expectations would also be helpful in allowing a smoother transition.

\section{Declaration of interest}

M.S., S.G. and G.S. moved to the UK from India under the International Fellowship Scheme.

Mitesh Shah Consultant Psychiatrist, Santanu Goswami Consultant Psychiatrist, Gagandeep Singh Consultant Psychiatrist, Mersey Care NHS Trust, Liverpool, *Robert Brown Consultant Psychiatrist, Rehabilitation Services, Adult Mental Health Directorate, Rathbone Hospital, Mill Lane, Liverpool L97JP, e-mail: marie.jones@merseycare.nhs.uk,

\title{
The International Fellowship Scheme for consultant psychiatrists: trainees' perspective
}

The NHS International Fellowship Scheme for consultants offers overseas consultants, in specialties including psychiatry, an opportunity to work in the UK (Goldberg, 2003). This was launched by the Department of Health in 2002 and so far over 100 consultant psychiatrists have been recruited. However, there are several aspects of the project that are unclear. How long will this recruitment continue? Are there any arrangements in place to encourage overseas consultants to return to their home country at the end of their fellowship? Are they eligible to train senior house officers (SHOs) and specialist registrars (SpRs)? Will the recruitment under the scheme have an impact on job opportunities for SpRs currently training in the UK? Why is membership of the Royal College of Psychiatrists being granted to the newly recruited consultants without an examination? These and many more concerns have arisen in the wake of this scheme. In this article, we evaluate the scheme, discuss its implications and suggest possible ways forward.

\section{Benefits of the scheme}

This scheme has a range of potential benefits. It will help to fill the vacant consultant posts in the National Health Service (NHS). Careful and selective recruitment of highly skilled and truly committed consultants can make a real difference to mental health service delivery.

The overseas consultants and potentially their home countries have as much to gain as the NHS. The scheme offers them an opportunity to work 'in a different health system, acquire new skills, get wider work experience, pursue research interests and develop their teaching skills' (Mellor, 2003). Individual NHS trusts are also providing many, less well-publicised, voluntary services in 
their home countries, in areas such as mental health and women's health (Mellor, 2003).

special articles

\section{Induction, mentoring and continuing professional development}

Most trusts have programmes to help overseas consultants familiarise themselves with the healthcare system and life in the UK in general. The trust which employs the consultants arranges for them to attend an induction programme, which includes an introduction to life in the UK, clinical aspects of the job and a discussion of cultural issues which may be unfamiliar. Most trusts also arrange training in the Mental Health Act 1983 and assist in obtaining Section 12(2) approval. However, there is a need to evaluate current induction programmes to explore whether they are uniform and fulfil both professional and pastoral needs.

A trust should also, in consultation with the consultant, appoint a medical colleague to act as mentor. If the post involves research, a research mentor should also be assigned. The mentor should discuss with the consultant their needs for continuing professional development (CPD) and opportunities for study leave and further training. A trust should also identify a member of staff to ensure pastoral support during the transition period. This should include an introduction to social and professional networks for the doctors and their families.

The Royal College of Psychiatrists has a significant role to play in providing advice on opportunities for CPD and in ensuring a supportive and comfortable working environment for the new consultants.

\section{Trainees' concerns}

A number of experts have expressed ethical and moral concerns about recruitment of consultant psychiatrists from low- and middle-income countries (Mellor, 2003; Patel, 2003; Ndetei et al, 2004). In addition, how does this scheme affect the training and career opportunities of UK trainees? There are two major issues that should be considered.

First, although the posts are advertised as nontraining appointments, this is not always the case. Many overseas consultants are being appointed to training posts and are closely involved in the training of SHOs and SpRs. There are several aspects of psychiatric training in the UK (e.g. preparing trainees for the MRCPsych examinations) that only experienced trainers can adequately cover. Fortunately, overseas consultants are usually highly skilled and have a wealth of clinical and research experience and, in general, it is only the form and not the content of postgraduate psychiatric training that tends to vary across countries. However, there is a clear case for a longer period before these consultants become trainers. There is also a need for tighter monitoring of the training process. The College has a key role to play in teaching these consultants to be good trainers (e.g. by organising training workshops). Once in the role of trainer, the consultants should undergo the same periodical review as existing UK psychiatrists.

Second, there is the issue of conferring the MRCPsych qualification on overseas consultants after a relatively short period of time. It is perceived, particularly by trainees, that some consultants recruited from overseas are granted Membership far too readily, thus causing resentment in those who have worked hard to obtain the qualification. The decision to confer Membership should only take place following extensive discussions with all the stakeholders. It could be argued that conferring some alternative qualification would be more appropriate. However, by conferring Membership the College could ensure CPD through College meetings and workshops, etc.

\section{Ways forward}

It is evident that there are no easy solutions to some of the pitfalls of the scheme. We therefore suggest some measures that might improve the effectiveness of the recruitment scheme.

\section{Prior to recruitment}

First, the scheme should be more selective and should assess the commitment and motivation of prospective recruits to ensure that they are coming to the UK for the right reasons. Second, there needs to be agreement on the number of consultants to be recruited and over what period of time. The number should be balanced against the number of specialist registrars who are likely to complete their training and qualify for consultant posts. Third, it needs to be ensured that a strictly ethical recruitment policy is followed by all NHS trusts and this should be closely monitored with tighter regulations in place to guarantee adherence. Finally, the scheme needs to be encouraged within the context of 'exchange programmes' and better support and advice should be available to the overseas consultants should they wish to return to their home countries.

\section{After recruitment}

Trusts need to provide more thorough induction programmes and adequate peer support and mentoring. There should also be a post-induction review to determine how well recruits are settling into their new roles, and whether they are experiencing any cultural or professional problems.

A much wider debate, including trainees, should discuss the eligibility of overseas consultants to become trainers and members of the College. The College should extend CPD opportunities to the recruits and support them in becoming good trainers for SHOs and SpRs.

\section{Long-term solutions}

Although the scheme represents a short-term solution to the problem of unfilled UK consultant posts in psychiatry, other long-term approaches should not be overlooked. 
These include increasing the intake into UK medical schools, encouraging more medical students into psychiatry and increasing the number of SHO and SpR training posts in psychiatry. In addition, current government initiatives such as Modernising Medical Careers will allow greater opportunities to experience psychiatry in Foundation Years 1 and 2.

\section{Conclusion}

In principle, the NHS International Fellowship Scheme has broad-ranging moral, logistic and training implications. There needs to be a much wider debate of the specific details of the programme and more intense monitoring of its effectiveness. Recognising and rectifying any failings early on will prevent it from becoming a chronic dysfunctional programme, delivering little benefit and compromising low- and middle-income countries.

\section{Declaration of interest}

S.K is a consultant psychiatrist from India who came to England under the NHS International Fellowship Scheme. S.G. and B.C. are SpRs training in the UK.

special articles

\section{References}

GOLDBERG, D. (2003) The NHS International Fellowship Scheme for Consultant Psychiatrists. Newsletter of the Faculty of General and Community Psychiatry, 6, 5-6.

MELLOR, D. (2003) Commentary: recruitment is ethical. BMJ, 327 928.

NDETEl, D., KARIM, S. \& MUBBAHAR, M. (2004) Recruitment of consultant psychiatrists from low- and middleincome countries. International Psychiatry, 6, 15-18.

PATEL,V. (2003) Recruiting doctors from poor countries: the great brain robbery. BMJ, 327, 926-928.

*Sanju George Specialist Registrar in General Adult Psychiatry, Sandwell OutreachTeam, 6-6A Simpson Street, Oldbury, Birmingham B69 4AL, e-mail: sanju.george@talk21.com, Bill Calthorpe Specialist Registrar in General Adult Psychiatry, Queen Elizabeth Psychiatric Hospital, Birmingham, Sudhir Khandelwal Consultant in General Adult Psychiatry, Glenfield Hospital, Leicester 